\title{
MAPping out breast cancer chemoprevention
}

$\mathrm{B}$ reast cancer is the second most common cause of death from cancer, and risk reduction measures are paramount to reduce the mortality associated with this disease. Estrogens are involved in normal breast development and data from numerous preclinical models have established that they induce breast tumors. Therefore, there has been an intensive focus on chemoprevention approaches to reduce the agonist effects of estrogen. Notably, the selective estrogen-receptor modulators, tamoxifen and raloxifene, have demonstrated dramatic risk-reduction effects in large randomized clinical trials. Tamoxifen reduces the number of invasive cancers in high-risk women by almost $50 \%$ and was shown to lower the incidence of breast cancer by $38 \%$ without affecting mortality. However, raloxifene and tamoxifen both increase the risk of endometrial cancer and increased risk of venous thromboembolism is associated with tamoxifen use, thereby limiting the use of these agents for chemoprevention.

Aromatase inhibitors also suppress estrogen levels in postmenopausal women, and a clinical trial comparing the aromatase inhibitor, letrozole, with tamoxifen demonstrated the superior efficacy of letrozole in reducing the development of contralateral breast tumors. The oral aromatase inhibitor exemestane has also demonstrated chemopreventive effects in adjuvant trials, making aromatase inhibitors the preferred treatment choice for women with early-stage disease. To address the efficacy of these preventative therapies, Paul Goss and collaborators conducted the NCIC Clinical Trials Group Mammary Prevention.3 study (NCIC CTG MAP.3) and the first results have been reported.

In this double-blinded, randomized, placebo-controlled trial, 4,560 healthy postmenopausal women at high risk for breast cancer (according to established risk criteria) were randomly assigned to receive either exemestane $(n=2,285)$ or

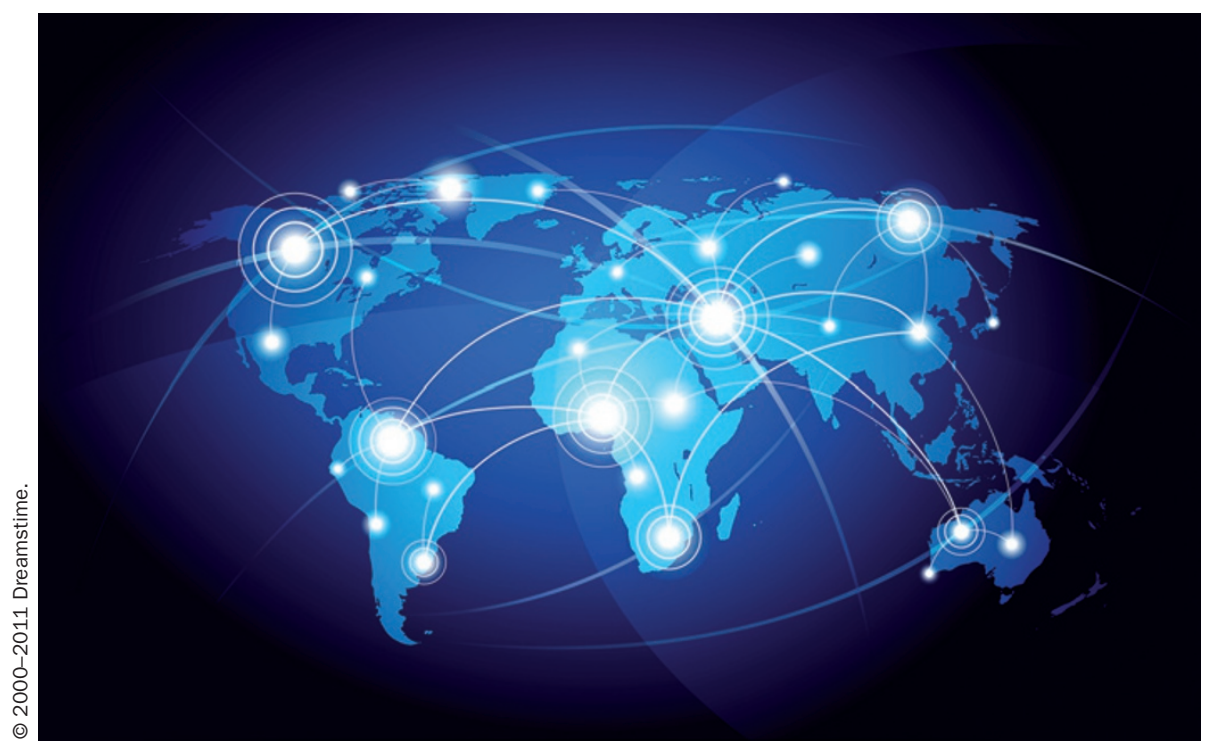

placebo $(n=2,275)$. At a median follow-up period of 35 months, 11 invasive cancers were detected in the exemestane arm compared with 32 in the placebo arm. Exemestane resulted in a $65 \%$ relative reduction of an invasive breast cancer diagnosis compared with placebo. No significant differences in cardiovascular events, skeletal fractures or mortality from any cause were noted between the two arms. Symptoms and all grade adverse events occurred in $88 \%$ of women in the exemestane group and $85 \%$ of women in the placebo group. Importantly, no serious adverse events or end-organ effects were attributed to exemestane treatment.

Menopausal symptoms including hot flashes, sweating, arthralgia, and fatigue were slightly more common in women taking exemestane. Vasomotor and sexual symptoms were also worse in exemestanetreated women; however, these symptoms did not seem to affect health-related quality of life compared with placebotreated patients. Although aromatase inhibitors are known to be associated with mild loss of bone mineral density, there was no excess of fractures as a result of exemestane treatment. Precursor lesions, such as ductal carcinoma in situ and lobular carcinoma in situ, were also reduced in women receiving exemestane.

Goss and his team conclude that "exemestane significantly reduced invasive breast cancers in postmenopausal women who were at moderately increased risk for breast cancer. During a median follow-up period of 3 years, exemestane was associated with no serious toxic effects and only minimal changes in health-related quality of life."

Some limitations of this trial include the low number of events, the relatively short median 3-year follow up, and the fact that the optimal duration of endocrine therapy is still unknown. In the postmenopausal breast cancer setting, clinicians now have three options for breast cancer chemoprevention-tamoxifen, raloxifene, and exemestane. When one drug should be used in preference over another is still unclear, although in women with increased venous thromboembolic risk, exemestane may be the preferred option.

\section{Lisa Hutchinson}

Original article Goss, P. E. et al. Exemestane for breastcancer prevention in postmenopausal women. N. Engl. J. Med. 364, 2381-2391 (2011) 